\title{
Atmospheric Turbulence Degraded Image Restoration Using Back Propagation Neural Network
}

\author{
Azad Singh $^{1}$ and Rajeev Kumar Singh ${ }^{2}$ \\ Department of Computer Science Engineering and Information Technology \\ Madhav Institute of Technology \& Science \\ Gwalior, India \\ 1azadsngh82@gmail.com, ${ }^{2}$ rajeev.mits1@gmail.com
}

\begin{abstract}
Atmospheric blur is the distortion of image due to long time exposure, fog, wind speed and due to randomly change in refractive index of air through which light travels. Atmospheric blur also occur through non-uniform geometric deformation of image. In this article, we propose a method for restoring atmospheric degraded image using artificial neural network. In proposed methodology use multilayer feed-forward network which trained by error back propagation algorithm and randomly initialize weights of network. This technique provides better result to restore atmospheric blur image and also in the presence of noise.
\end{abstract}

Keywords- Back Propagation, ANN (Artificial Neural Network), atmospheric turbulence, PSNR (Peak Signal to Noise Ratio)

\section{Introduction}

Atmospheric blur degrades the image due to variety of factors like long-time exposure, wind speed and due to temperature. In earth's atmosphere dust particles and aerosols revolving that reflects the light before reaches to camera lens. Images can be degraded due to fluctuation in the refractive index of atmosphere [1]. The images may be affected due to temperatures i.e. winter and summer season. In winter, haze or fog effect occurs thus the visibility of scene is not so clear and get degraded image [7]. We often seen mirage when capturing image at hot places or summer season.

The degradation model of imagine system can be define as,

$g_{k}=I_{k} \otimes h+n_{k}$

Where, $g_{k}$ is the degraded image, in which $\mathbb{Q}$ denotes the two-dimensional linear convolution operation, $I_{k}$ is the original image and $h$ is point spread function $n_{k}$ is the additive noise [3]. For study of turbulence effect, we will consider scenario of stars; the effect of turbulence at a given moment is to split the images of star into multiple speckles that create jitter around as the atmosphere changes. Each speckles is about the size of diffraction limited PSF (Point Spread Function) [5], [9]. The blurring of images causes two problems: 1) Light from closely separated objects is blended together and objects may not be recognized as separable, this is known as confusion. 2) Light from a single object is spread over a larger angular area making it tough to detect against noisy background. The effects to atmospheric turbulence can be measured by calculating the scintillation index. This index is related to the standard deviation and mean of the intensity distribution in an image [2].

The point spread function of atmospheric turbulence image can be described using a Gaussian function. 


$$
h\left(x, y, \sigma_{G}\right)=C \exp \left(\frac{x^{2}+y^{2}}{2 \sigma^{2} G}\right)
$$

Here, $\sigma_{G}$ determines the quantity of spread of the blur in image, and the constant $C$ is to be chosen so that above equation is satisfied [4].

In this paper we used feed forward neural network to restore image using first order feature of image i.e., mean value of each pixel [6]. This method is capable to handle both spatially varying blur and shift-invariant atmospheric blur images.

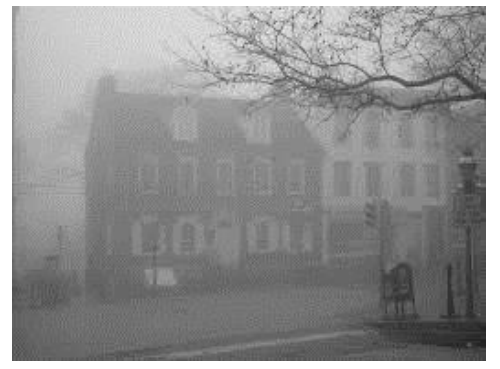

(a) Foggy blur image

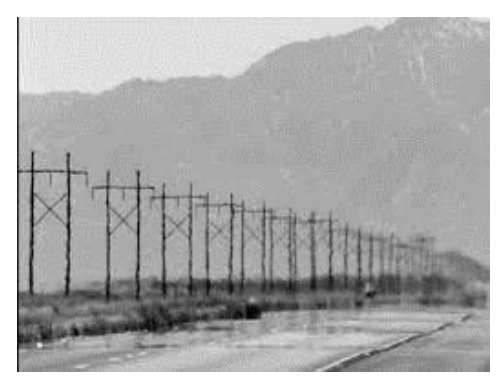

(b) Image is blurred due to temperature

Figure 1.

\section{Artificial Neural Network}

Artificial neural network is an information paradigm which is inspired by our biological nervous system, like brain and processing system. In which artificial neurons are interconnected to each other through weights [14], [16]. It can be trained through the supervised learning process.

\section{It can be classified into two types:}

- Simple neural network

- Fuzzy neural network

\section{Simple Neural Network}

The simple neural network is a two layers or multilayer feed-forward and feedback network with $\mathrm{M}$ input and $\mathrm{N}$ output units. Each input unit is connected to each of the output units, and each connection is associated with weight, which are denoted as $w_{1}, w_{2} \ldots w_{N}$ respectively or the strength of the connection. The inputs and the weights are real values [10]. A negative value for a weight indicates an inhibitory connection while a positive value indicates an excitatory one. Although in biological neurons, has a negative value, it may be assigned a positive value in artificial neuron models. The output unit is assigned with activation through which modelled the output of network. The Figure2 shows architecture of multilayer feed-forward network.

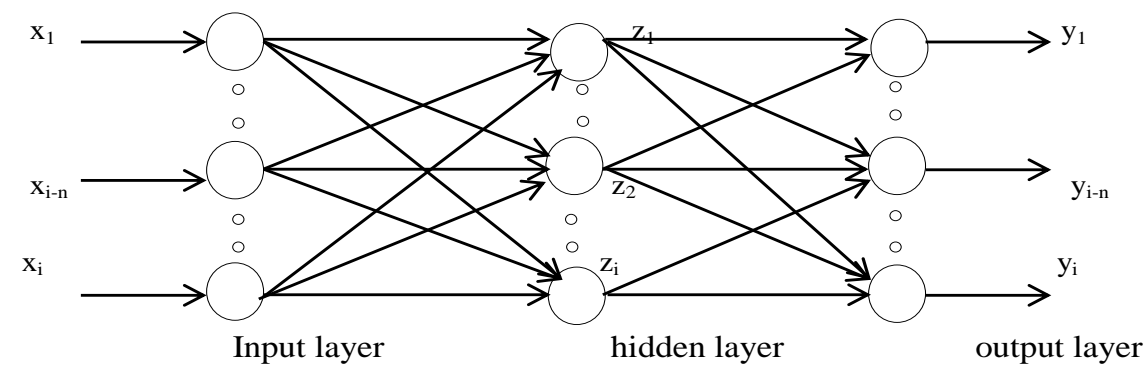

Figure 2. Architecture of Multilayer Feed- Forward Network 
A negative value for a weight indicates an inhibitory connection while a positive value indicates an excitatory one. Although in biological neurons, has a negative value, it may be assigned a positive value in artificial neuron models. The output unit is assigned with activation through which modelled the output of network.

\section{Fuzzy Neural Network}

A fuzzy neural network or neuro-fuzzy system is a combination of neural network and fuzzy logic. Neural network have advantages in the area of learning, classification and optimization whereas fuzzy logic has advantages in area such as reasoning on a high level i.e., semantic or linguistic [12]. This network can work on realistic problem of world where the information is incomplete, wrong or contradictory and this process may be time consuming and error prone.

Neuro-fuzzy system combines the advantages of fuzzy systems, which deal with explicit knowledge, which can be explained and understood, and neural network, which deal implicit knowledge which can be acquired by learning [10], [13]. Neural network learning provides a good way to adjust the expert's knowledge and automatically generate additional fuzzy rules and membership functions, to meet certain specification. The neural network advantages has own, i.e., adapt to unknown situation, robustness, autonomous learning. Where, fuzzy logic enhances the generalization capability of network system by providing more reliable output when extrapolation is needed beyond the limits of training data The neural network and fuzzy has own advantages and disadvantages but by combination of both may completely disappear the disadvantages[15]. The structure of neuro-fuzzy system is shown in figure given below:

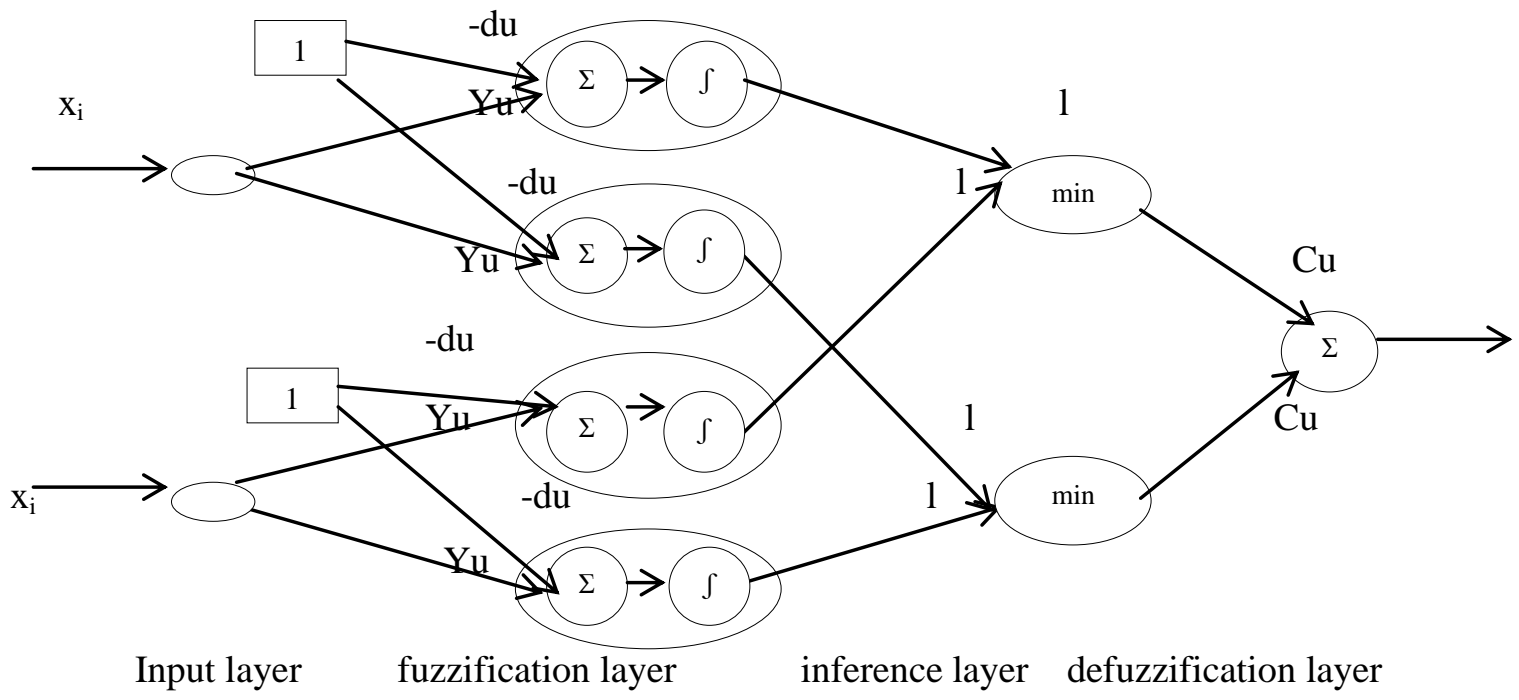

\section{Figure 3. Architecture of Neuro-Fuzzy System and this System has Two Input, One Output and Two Rules}

\section{Back Propagation Algorithm}

The error back propagation algorithm is used for training multilayer feed-forward neural network, so that the network can be trained to capture the mapping implicit in the given input and output matrix or pattern pairs. The back propagation generalized the least square algorithm that modifies network weights to minimize the mean square error between desired and actual output [8]. The back propagation algorithm falls into the general category of gradient descent algorithms based delta-learning rule, which intend to find the minima/maxima of a function by iteratively moving in the direction of the 
negative of the slope of the function to be minimized /maximized. The network is trained by supervised learning. The error function is given as the sum of the square of difference between target values to output values.

$e=\frac{1}{2}(d-k)^{2}$

Where, $e$ is the error value, $d$ is the desired value and $k$ is the observed value at output layer.

In this algorithm, the weights are initializing with some small random values. In the feed forward network each input unit $\left(X_{i}\right)$ receives an input signal and transmits this signal to each of the hidden layers $z_{1}, z_{2} z_{3} \ldots z_{p}$. Then each hidden layer calculates the activation functions and sends its signal to each output unit. The output unit calculates the activation function to form the response of net value of the given input signal or pattern [11]. Then each output unit compares its computed activation with its target value $t_{k}$ to determine the associated error for that input pattern with that unit. Based on error, the factor $\delta_{k}(k=1, \ldots, m)$ is calculated and used to distribute error at output unit $y_{k}$ back to all units in the previous layer. Similarly, the error factor $\delta_{p}(k=1, \ldots, p)$ is computed at each hidden layer $z_{j}$. So that weights are updated using the factor $\delta$ and activation function.

A gradient descent strategy is adopted to minimize the error. The chain rule for differentiation turns out to be

$\frac{\partial E(n)}{\partial w_{j k}(n)}=\frac{\partial E(n)}{\partial s_{j}(n)} \frac{\partial s_{j}(n)}{\partial y_{j}(n)} \frac{\partial y_{j}(n)}{\partial v_{j}(n)} \frac{\partial v_{j}(n)}{\partial w_{j k}(n)}$

This can be simplified into,

$\frac{\partial E(n)}{\partial w_{j k}(n)}=-s_{j}(n) \hat{f}_{j}\left(v_{j}(n)\right) y_{j}(n)$

The final rule for updating weights becomes,

$\Delta w_{j k}(n)=\alpha \delta_{j}(n) y_{j}(n)$

Where,

$$
\begin{gathered}
\delta_{j}(n)=-\frac{\partial E(n)}{\partial v_{j}(n)} \\
=-\frac{\partial E(n)}{\partial s_{j}(n)} \frac{\partial s_{j}(n)}{\partial y_{j}(n)} \frac{\partial y_{j}(n)}{\partial v_{j}(n)} \\
=s_{j}(n) f_{j}\left(v_{j}(n)\right)
\end{gathered}
$$

for the last layer and

$$
\delta_{j}(n)=\tilde{f}_{j}\left(v_{j}(n)\right) \sum_{k} \delta_{i}(n) w_{i j}(n)
$$

for the intermediate hidden layers.

We use the batch learning scheme for weight updating. All the training vector assign to the network and the change in all weights is computed from each input vector. Then at the end we update the weights according to the sum of all updates. Thus the weight update is only performed after every epoch.

If $p=$ pattern in one epoch, then 


$$
\Delta w=\frac{1}{p} \sum_{p=1}^{\infty} \Delta w_{p}
$$

\section{Neural Network Model Features}

A. Activation Function

The user also has an option of three activation functions for the neurons:

Unipolar sigmoid:

$$
f(x)=\frac{1}{1+e^{-2 x}}
$$

Bipolar sigmoid:

$$
f(x)=\frac{2}{1+e^{-\lambda x}}-1
$$

Tan hyperbolic:

$$
f(x)=\frac{e^{\lambda x}-e^{-\lambda x}}{e^{\lambda x}+e^{-\lambda x}}
$$

Radial basis function:

$$
f(x)=\frac{1}{\sqrt{2 \pi} \sigma} e^{-(x-\mu)^{2} / 2 \sigma^{2}}
$$

This activation functions are common to all the neurons in the ANN.

\section{A. Hidden Layers and Nodes}

We train feed forward network to restore blur image. It has an arbitrary number of hidden layers and hidden nodes, so that user can decide according to situation of blurry images.

\section{B. Stopping Criterion}

The rate of convergence for the back propagation algorithm can be controlled by the learning rate $\alpha$. A larger value of $\alpha$ would ensure faster convergence, however it may cause the algorithm to oscillate around the minima, whereas a smaller value of $\alpha$ would cause the convergence to be very slow [17].We need to have some stopping criterion for the algorithm as well, to ensure that it does not run forever. For our experiments, we use a three-fold stopping criterion. The back propagation algorithm stops if any of the following conditions are met:

- The change in error from one iteration to the next falls below a threshold that the user can set.

- The error value begins to increase. There is a relaxation factor here as well that allows minimal increase as it is also observed that the error tends to increase by small amount and then decrease again.

- If the number of iterations (or epochs) goes beyond a certain limit. In our case the limit is set to 300 .

\section{Momentum Factor}

The main purpose of the momentum factor is to accelerate the convergence of error back propagation algorithm. This method makes the current weight adjustment with a fraction of the previous weight adjustment. It is found that momentum allows the network to adjust large weight as long as the correction proceeds in the same direction of several patterns. By using momentum the network does not proceed in the direction of gradient, 
but travels in the direction of combination of the current direction and the previous direction where the weight updating is made.

The weight updating for back propagation with the momentum is given as,

$$
\begin{aligned}
& w_{j k}(t+1)=w_{j k}(t)+\alpha \delta_{k} z_{j}+\mu\left[w_{j k}(t)-w_{j k}(t-1)\right] \\
& v_{j k}(t+1)=v_{j k}(t)+\alpha \delta_{j} z_{i}+\mu\left\lfloor v_{i j}(t)-v_{i j}(t-1)\right]
\end{aligned}
$$

$\mu$ is called the momentum factor and $\alpha$ is the learning rate. It ranges from $0<\mu<1$.

\section{Proposed Methodology}

For the successfully deployment of our proposed work, firstly, we take input of original image for training the neural network. The pre-processing process has to perform on image before training of network. In pre-processing process, we will normalize the size of image into $n \times n$ blocks and also normalize pixel value of image by converting image into double integer type and divided by 255 . We have to reshape the image from 2-D to 1-D as input data in neural network. Further, a new feed-forward network of 20 hidden layer and one output layer is designed. The activation function will be associated with each layer of network described above and randomly initializes the weights of network. Then, Take input of blurred image and pre-process image as above discussed.

After the above process, neural network will compute the error of image by calculating the MSE (Mean Square Error). Then we will set the parameter of back propagation algorithm to train the network according to original image. After, each iteration weights are updated and compute error. We use the batch learning scheme for weight updating. All the training vector assign to the network and the change in all weights is computed from each input vector. Then at the end we update the weights according to the sum of all updates. Thus the weight update is only performed after every epoch. If error is acceptable after validation check then stop the network and save weights of network. The validation check is used to prevent network from stuck in local minima. Get restored image after simulating the network and convert from 1-D to 2-D.

The following steps are performed given below:

Step-1. Take input of original image $I(x, y)$.

Step-2. Normalize size of image $I(x, y)$ into $n \times n$ blocks.

Step-3. Normalize image $I(x, y)$ by dividing with 255 .

Step-4. $\quad$ Take input blurred image $B(x, y)$ and perform Step 2 and Step 3.

Step-5. Reshape input and blurred image from 2-D* to 1-D*.

Step-6. Create a new feed-forward neural network 20 hidden layer and one output layer. The tangent sigmoidal and uniform activation function is associated with each hidden and output layer respectively.

Step-7. Randomly initializes weight of network to train network by back propagation algorithm and set parameter for stop the training.

Step-8. Apply blurred image to trained network to get restored image.

Step-9. $\quad$ Reshape image data from 1-D* to 2-D*.

Step-10. Finally get restored image.

The block diagram of proposed method is shown in Figure 4. In which we train feed forward neural network will train through using back propagation algorithm. Original 
image is used to train network and adjust weights of network to get output image same as original image. Then weights of network will be saved. After it apply blurred image to trained network to get restored image. Then calculate mean square error of restored image.

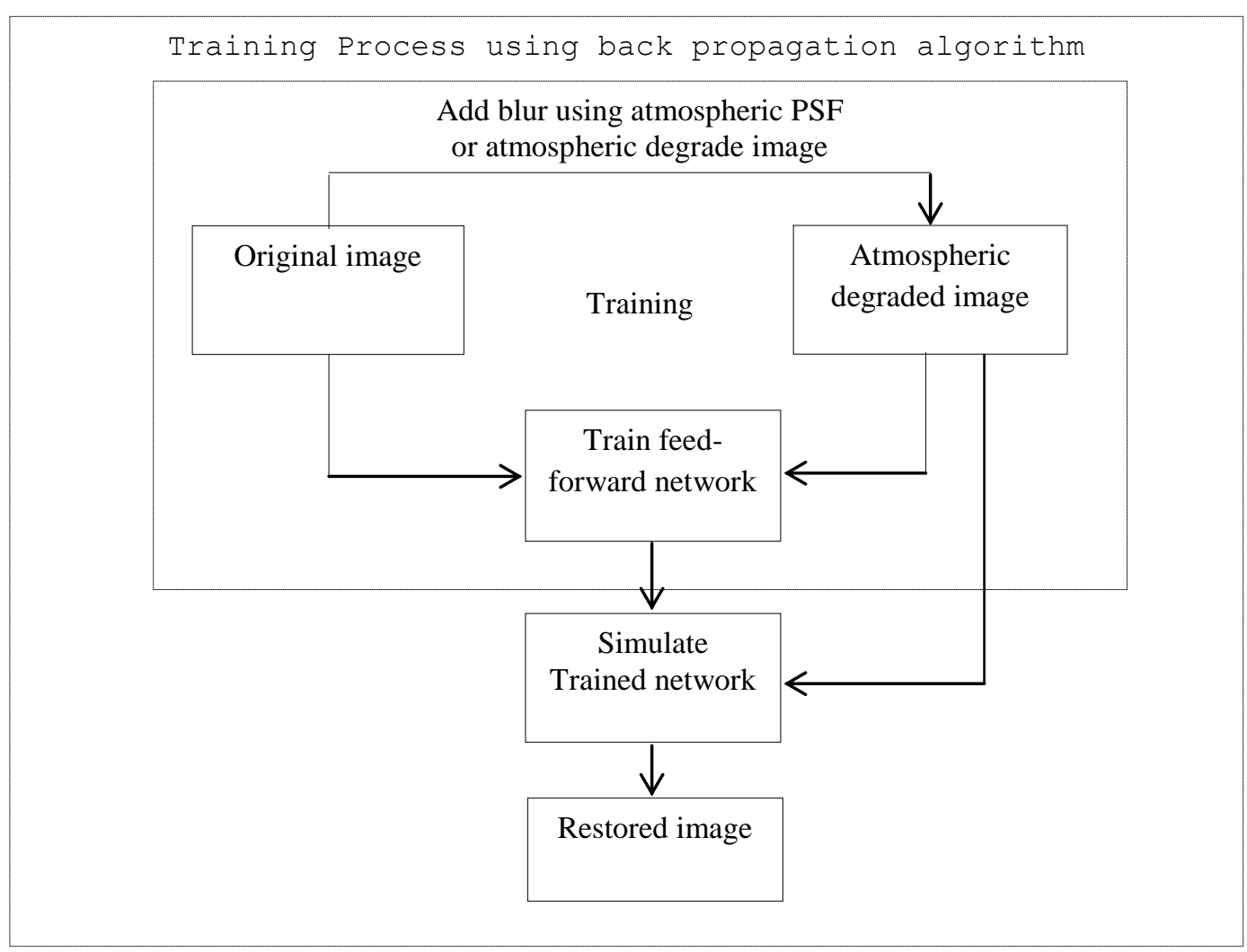

Figure 4. The Block Diagram of our Proposed Method

We train the network using back propagation algorithm and the flow chart of algorithm shown in Figure 5. 


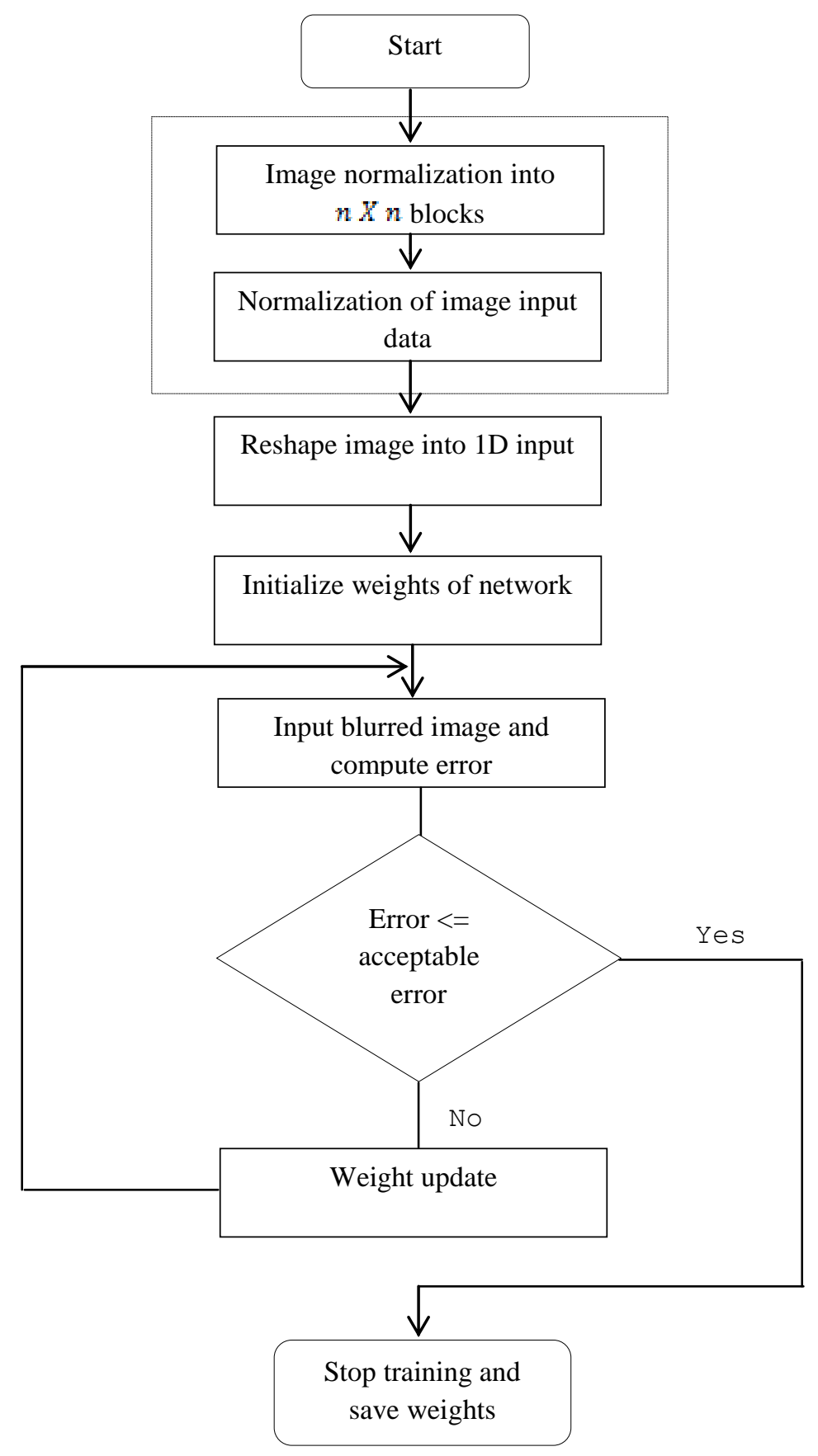

Figure 5. Flow Chart Diagram of Back Propagation Algorithm

\section{Result Analysis}

We perform restoration process using our proposed methodology on four images and get the result. The result is given below: 
Table 1.

\begin{tabular}{|l|l|l|l|l|}
\hline S.no & Name of image & $\begin{array}{l}\text { PSNR Value } \\
\text { of blurred } \\
\text { image }\end{array}$ & $\begin{array}{l}\text { PSNR Value } \\
\text { of Restored } \\
\text { image }\end{array}$ & $\begin{array}{l}\text { SSIM value } \\
\text { of restored } \\
\text { image }\end{array}$ \\
\hline 1 & Foggy image & 58.84 & 68.77 & 0.76 \\
\hline 2 & $\begin{array}{l}\text { Satelite } \\
\text { image }\end{array}$ & 76.54 & 82.01 & 0.93 \\
\hline 3 & Moon image & 69.19 & 74.60 & 0.66 \\
\hline
\end{tabular}

Compare the PSNR value of restored image using our proposed methodology to other existing techniques

Table 2.

\begin{tabular}{|l|l|l|l|l|l|}
\hline S.no & $\begin{array}{l}\text { Name of } \\
\text { Image }\end{array}$ & $\begin{array}{l}\text { PSNR } \\
\text { Value } \\
\text { of } \\
\text { blurred } \\
\text { image }\end{array}$ & $\begin{array}{l}\text { PSNR value } \\
\text { of restored } \\
\text { image using } \\
\text { kurtosis[1] }\end{array}$ & $\begin{array}{l}\text { PSNR } \\
\text { value of } \\
\text { restored } \\
\text { image } \\
\text { using } \\
\text { PCA [6] }\end{array}$ & $\begin{array}{l}\text { PSNR value } \\
\text { of restored } \\
\text { image using } \\
\text { proposed } \\
\text { methodology }\end{array}$ \\
\hline 1 & $\begin{array}{l}\text { Foggy } \\
\text { image }\end{array}$ & 58.84 & 59.54 & 63.54 & 68.77 \\
\hline 2 & $\begin{array}{l}\text { Satelite } \\
\text { image }\end{array}$ & 76.54 & 78.89 & 79.69 & 82.01 \\
\hline 3 & $\begin{array}{l}\text { Moon } \\
\text { image }\end{array}$ & 69.19 & 71.44 & 72.43 & 74.60 \\
\hline
\end{tabular}

The result get by using proposed methodology is comapered with other existing methodology. By analysing the result we have found our methodolgy provide better or acceptable result.

Similarly, perform test on noisy image, to check robustness of proposed method in the presence of noise. We add noise impulse noise in an image. Now train the network for noisy images and perform several iterations to minimize the error of image. After the training network simulate the network for noisy and restored image, and then calculate the PSNR value of restored image. The PSNR values are given below:

Table 3.

\begin{tabular}{|l|l|l|l|l|l|}
\hline S.no & $\begin{array}{l}\text { Name of } \\
\text { Image }\end{array}$ & $\begin{array}{l}\text { PSNR } \\
\text { Value } \\
\text { of } \\
\text { blurred } \\
\text { image }\end{array}$ & $\begin{array}{l}\text { PSNR value } \\
\text { of restored } \\
\text { image using } \\
\text { kurtosis [45] }\end{array}$ & $\begin{array}{l}\text { PSNR } \\
\text { value of } \\
\text { restored } \\
\text { image } \\
\text { using } \\
\text { PCA [50] }\end{array}$ & $\begin{array}{l}\text { PSNR value } \\
\text { of restored } \\
\text { image using } \\
\text { proposed } \\
\text { methodology }\end{array}$ \\
\hline 1 & $\begin{array}{l}\text { Foggy } \\
\text { image }\end{array}$ & 56.91 & 58.89 & 61.24 & 67.57 \\
\hline 2 & $\begin{array}{l}\text { Satellite } \\
\text { image }\end{array}$ & 59.578 & 65.81 & 71.79 & 77.154 \\
\hline 3 & $\begin{array}{l}\text { Moon } \\
\text { image }\end{array}$ & 60.47 & 66.84 & 70.32 & 73.324 \\
\hline
\end{tabular}


We have taken three different types of atmospheric turbulence images like foggy image, satellite image and moon image then restored these images by different techniques and result shown below:

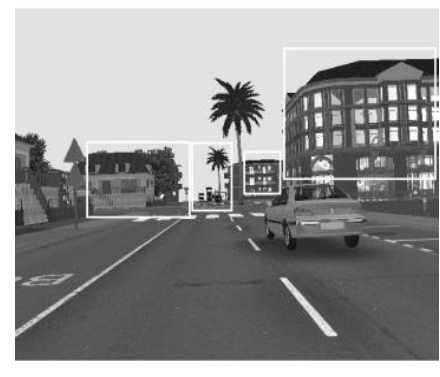

(a) original image

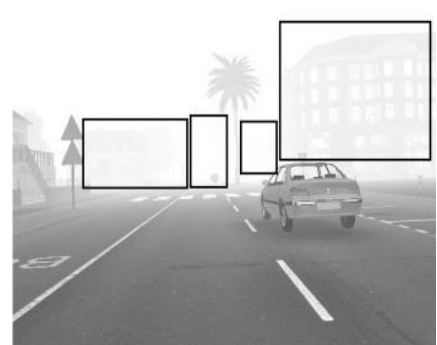

(b) foggy image

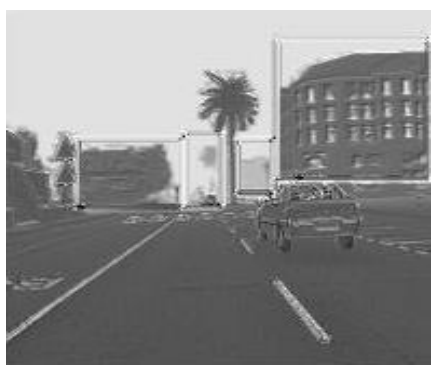

(c) Restored image Using PCA

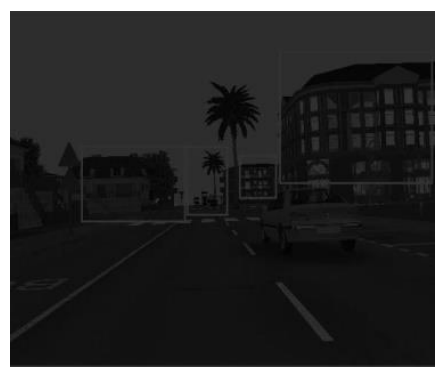

(d) Restored image using proposed methodology

Figure 6

Example of Satellite image:

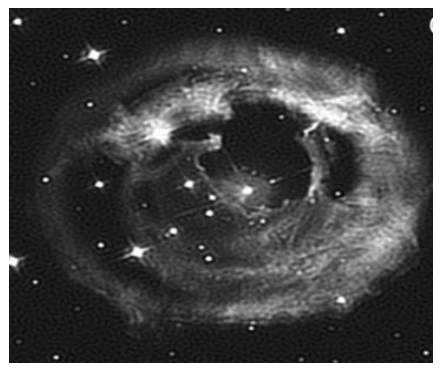

(a) original image

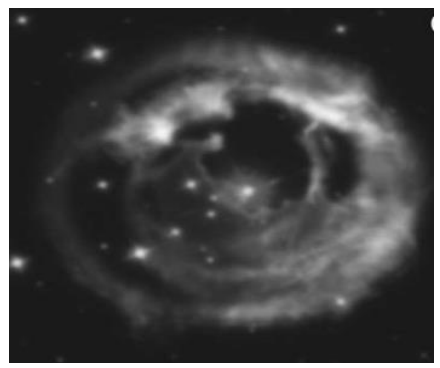

(b) blurred satellite image kurtosis

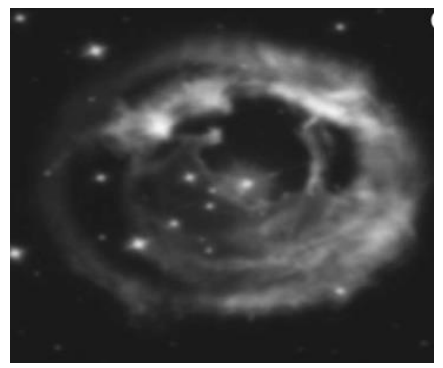

(c) Restored image using
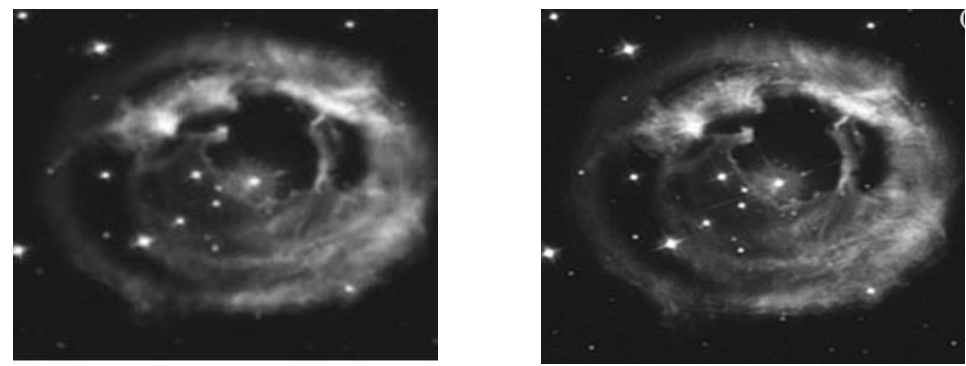

(d) Restored image using PCA

(e) Restored image using proposed methodology

Figure 7. 
Example of moon image:

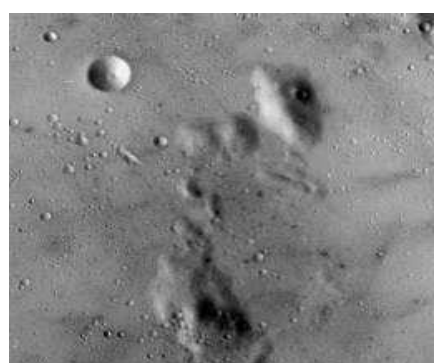

Figure 8 (a) original image

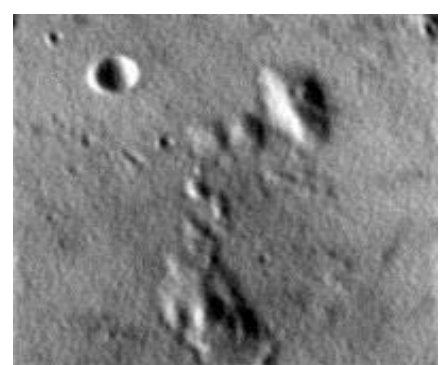

(b) moon blurred image kurtosis

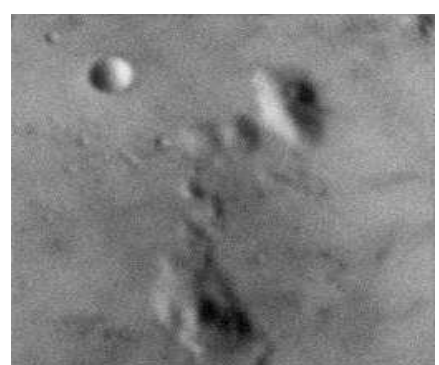

(c) Restored image using
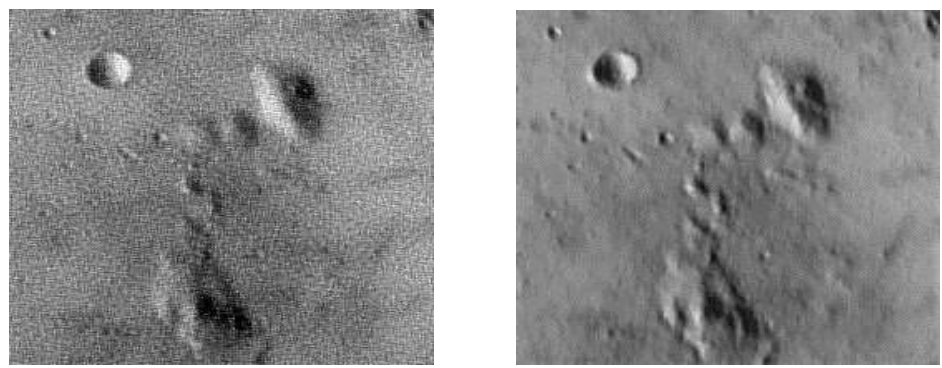

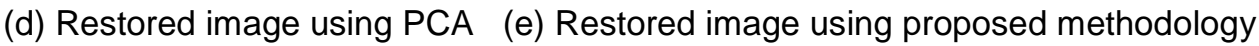

Now Figure 9 shows graph of comparison between the PSNR values of restored image as shown. The PSNR is peak signal to noise ratio is defined as

$$
\text { PSNR }=10 \log _{10} \frac{\sum_{i=1}^{M} \sum_{j=1}^{M} 255^{2}}{\sum_{i=1}^{M} \sum_{j=1}^{N}(f(i, j)-\hat{f}(i, j))^{2}}
$$

Where $\mathrm{Mx} \mathrm{N}$ is size of image, $\hat{f}(i, j)$ is restored image and $f(i, j)$ is original image.

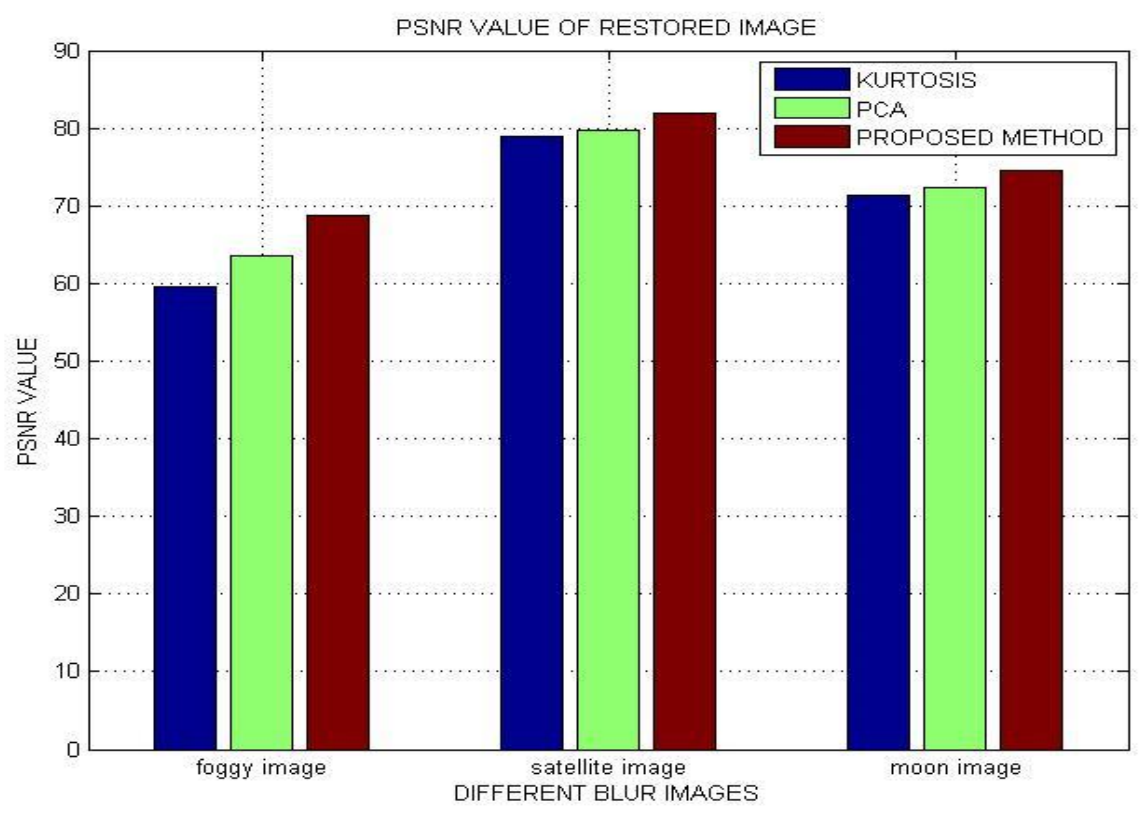

Figure 9. The Bar Graph of Comparison of Result 


\section{Conclusion and Future Scope}

Atmospheric restoration technique is depth field in which a large scope to do research work and have to increase efficiency of existing algorithm. In this paper we proposed a method for restoring atmospheric degraded image using neural network. We described about neural network and training algorithm by which train the network and also discuss first order feature by which initialize the weights of network. The results obtained by our methodology are acceptable and better than other existing techniques.

The work can be extended in future by using fuzzy neural network and genetic algorithm to get much better result than existing method.

\section{References}

[1] D. Li and S. Simske, "Atmospheric Turbulence Degraded Image by Kurtosis Minimization", IEEE Geoscience and Remote Sensing Letters, (2008) December.

[2] R. E. Hufnagel and N. R. Stanley, "Modulation transfer function associated with image through turbulence media”, J. opt. Soc. Amer: A, Opt. Image Sci., vol. 54, no. 1, (1964), pp. 52-61.

[3] G. M. Gluckman, "Kurtosis and the Phase Structure of Images", in 3rd International Workshop on Statistical and Computational Theories of Vision, Nice, France, (in conjunction with ICCV '03), Nice, France, (2003) October, pp. 12-15.

[4] L. Yan, M. Jin, H. Fang, H. Liu and T. Zhang, "Atmospheric Turbulence-Degraded Astronomical Image Restoration by Minimizing Second-Order Central moment", IEEE Geoscience And Remote Sensing Letters, vol. 9, no. 4, (2012) July, pp. 672-676.

[5] M. K. Hu, "Visual pattern recognition by moment invariants", IRE Trans. Inf. Theory, vol. 8, no. 2, (1962) February, pp. 179-187.

[6] D. Li, R. M. Mersereau and S. Simske, "Atmospheric Turbulence-Degraded Image Restoration Using Principal Component Analysis", IEEE Geoscience And Remote Sensing Letters, vol. 4, no. 3, July (2012), pp. 340-344.

[7] J.-B. Wang, N. He, L.-L. Zhang and K. Lu, "Single Image dehazing with a physical model and dark channel prior", Elsevier, neurocomputing, (2014) August.

[8] J. E. Dayhoff and J. M. DeLeo, "Artificial neural networks: Opening the black box", Cancer, vol. 91 (8 Suppl.) (2001), pp. 1615-1635.

[9] W. Zhou, A. C. Bovik, H. R. Sheikh and E. P. A. S. E. P. Simon celli, "Image quality assessment: from error visibility to structural similarity", Image Processing, IEEE Transactions on, vol. 13, (2004), pp. 600-612.

[10] R. J. T. Morris, L. D. Rubin and H. Tirri, "Neural network techniques for object orientation detection: solution by optimal feed-forward network and learning vector quantization approaches", IEEE Trans. Pattern Anal. Mach. Intell., vol. 12, no. 11, (1990), pp. 1107-1115.

[11] Y. L. Sun and S. Yu, "Improvement on performance of modified Hopfield neural network for image restoration", IEEE Trans. Image Process, vol. 4, no. 5, (1995), pp. 683-692.

[12] S. S. Young, P. D. Scott and N. M. Nasrabadi, "Object recognition using multilayer Hopfield neural network", IEEE Trans. Image Process, vol. 6, no. 3, (1997), pp. 357-372.

[13] L. O. Hall, A. M. Bensaid and L. P. Clarke, "A comparison of neural network and fuzzy clustering techniques in segmenting magnetic resonance images of the brain", IEEE Trans. Neural Networks, vol. 3, no. 5, (1992) pp. 672-682.

[14] C. C. Lee and J. P. Degyvez, "Color image processing in a cellular neural-network environment", IEEE Trans. Neural Networks, vol. 7, no. 5, (1996), pp. 1086-1098.

[15] F. Hussain and J. Jeong, "Visibility enhancement of scene images degraded by foggy weather conditions with deep neural networks", Hindawi publishing corporation journal of sensors, (2015) May.

[16] P. Baldi and J. Hornik, "Neural networks and principal component analysis: learning from examples without local minima", Neural Networks, vol. 2, no. 1, (1989), pp. 53-58.

[17] J. K. Paik and A. K. Katsaggelos, "Image restoration using a modified Hopfield network", IEEE Trans. Image Process, vol. 1, no. 1, (1992), pp. 49-63.

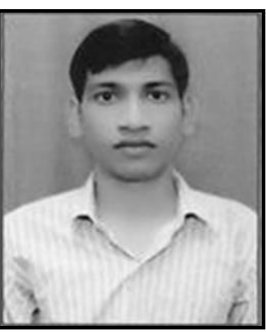

\section{Authors}

Azad Singh has received the B.E. degree in Information Technology, from Maharana Pratap College of Technology, Gwalior, India in 2012. Currently he is pursuing M.Tech. (Information Technology) from MITS, Gwalior. His research area includes Image restoration. 


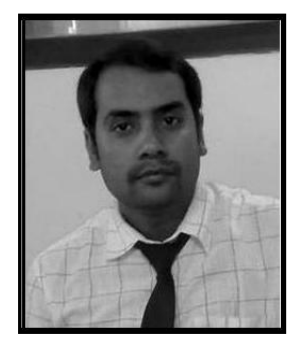

Prof. Rajeev Kumar Singh is working as assistant Professor, Department of Computer Science Engineering \& Information Technology, MITS, Gwalior, India. His passion is to contribute in research activities of Science \& Technology. His teaching and research include Image Processing and software engineering. 
International Journal of Signal Processing, Image Processing and Pattern Recognition Vol.8, No.12 (2015) 